\title{
Regarding the Case of Postpartum Sudden Cardiac Death After Spontaneous Coronary Artery Dissection in a Patient With Fibromuscular Dysplasia
}

\author{
Brandon W. Calenda ${ }^{a}$, b Jeffrey W. Olin ${ }^{a}$
}

\section{To the Editor}

We read with great interest the recent case report by Shoja and colleagues [1]. The authors describe a case of postpartum sudden cardiac death, with angiography and intravascular ultrasound (IVUS) demonstrating a spontaneous coronary artery dissection (SCAD) in the left main coronary artery. The authors go on to diagnose the patient with fibromuscular dysplasia (FMD) on the basis of focal dissection in the left vertebral artery on magnetic resonance angiogram (MRA). While FMD is common in patients presenting with SCAD [2], this patient cannot be diagnosed with FMD based on the available information.

In the United States Registry for Fibromuscular Dysplasia and in the French ARCADIA (Assessment of Renal and Cervical Artery Dysplasia) Registry, the diagnosis of FMD requires the radiographic detection of multifocal FMD ("string of beads") or focal, non-atherosclerotic stenosis in a non-dissected artery [3]. This patient's MRA apparently did not reveal multifocal or focal stenosis in any vascular bed. While these phenotypic presentations (SCAD and cervical artery dissection) are similar to patients with FMD, and in all likelihood genetically similar as well $[4,5]$, the absence of a focal stenosis or the "string of beads" appearance precludes the diagnosis of FMD.

\section{References}

1. Shoja T, Basman C, Jain S, Mangla A, Lasic Z. Postpartum sudden cardiac death after spontaneous coronary artery dissection in a patient with fibromuscular dysplasia.

Manuscript submitted January 22, 2018, accepted February 16, 2018

aVascular Medicine and Vascular Diagnostic Laboratory, The Zena and Michael A. Wiener Cardiovascular Institute and Marie-Josee and Henry R. Kravis Center for Cardiovascular Health, Icahn School of Medicine at Mount Sinai, One Gustave L. Levy Pl, Box 1033, New York, NY 10029, USA

${ }^{\mathrm{b}}$ Corresponding Author: Brandon W. Calenda, Vascular Medicine and Vascular Diagnostic Laboratory, The Zena and Michael A. Wiener Cardiovascular Institute and Marie-Josee and Henry R. Kravis Center for Cardiovascular Health, Icahn School of Medicine at Mount Sinai, One Gustave L. Levy P1, Box 1033, New York, NY 10029, USA. Email: Brandon.Calenda@mountsinai.org

doi: https://doi.org/10.14740/cr587wl
Cardiol Res. 2017;8(6):327-330.

2. Saw J, Ricci D, Starovoytov A, Fox R, Buller CE. Spontaneous coronary artery dissection: prevalence of predisposing conditions including fibromuscular dysplasia in a tertiary center cohort. JACC Cardiovasc Interv. 2013;6(1):44-52.

3. Olin JW. Expanding clinical phenotype of fibromuscular dysplasia. Hypertension. 2017;70(3):488-489.

4. Kiando SR, Tucker NR, Castro-Vega LJ, Katz A, D'Escamard V, Treard C, Fraher D, et al. PHACTR1 is a genetic susceptibility locus for fibromuscular dysplasia supporting its complex genetic pattern of inheritance. PLoS Genet. 2016;12(10):e1006367.

5. Debette S, Kamatani Y, Metso TM, Kloss M, Chauhan G, Engelter ST, Pezzini A, et al. Common variation in PHACTR1 is associated with susceptibility to cervical artery dissection. Nat Genet. 2015;47(1):78-83.

\section{Reply From Dr. Tannaz Shoja et al}

We thank Drs. Calenda and Olin for their interest in our case report [1]. As they state, the diagnosis of fibromuscular dysplasia (FMD) in a patient with spontaneous coronary artery dissection (SCAD) cannot be made based on the angiographic documentation of isolated vertebral artery dissection according to the United States Registry for Fibromuscular Dysplasia and French ARCADIA (Assessment of Renal and Cervical Artery Dysplasia) Registry [2].

To clarify, on initial presentation, the patient we described had multifocal dissections in left vertebral artery $(\mathrm{C} 2 / 3$ and C6 level), best visualized with time-of-flight technique (arrows in Fig. 1), while gadolinium-enhanced imaging suggested stenosis in the left vertebral artery, and also multifocal regions of irregularity/narrowing in the right vertebral artery best visible on gadolinium-enhanced imaging (arrows in Fig. 2). Our patient would be classified as multiple vascular bed involvement on the basis of the above-mentioned findings. Follow-up imaging 15 months after the initial presentation (performed after submission of the case report) showed healed dissection areas in the left vertebral artery with worsening diffuse narrowing in the right vertebral artery (arrows in Fig. 3). Patient discontinued all prescribed medications except ASA, between 4 and 15 


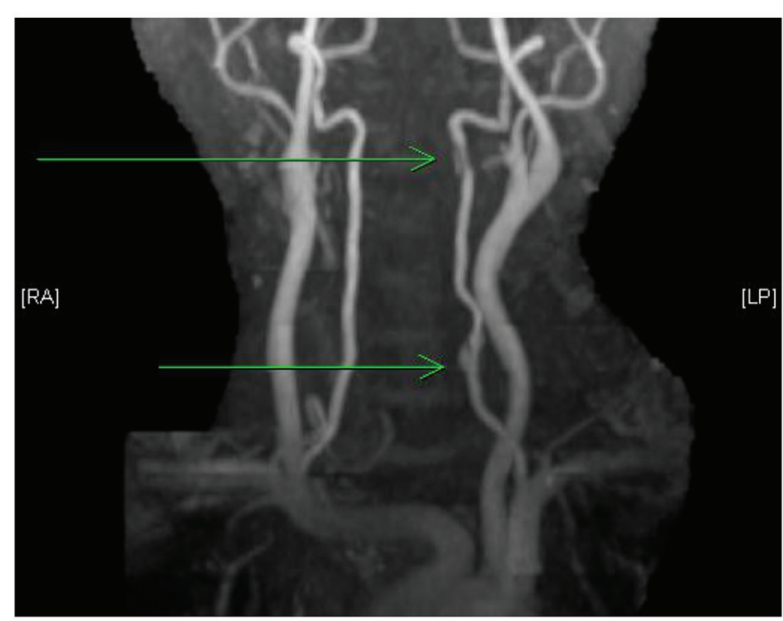

Figure 1. Multifocal dissection in left vertebral artery $(C 2 / 3$ and $C 6$ levels).

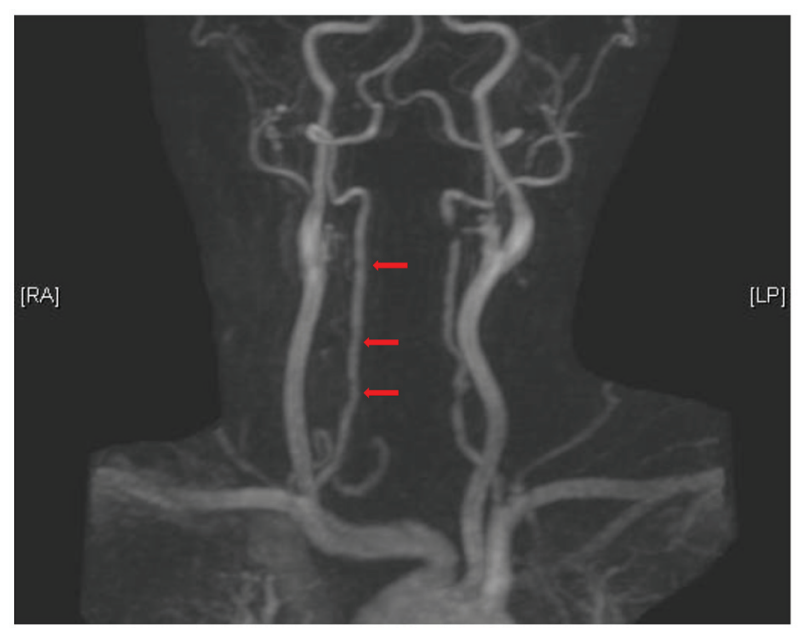

Figure 2. Stenosis in left vertebral artery and also multifocal regions of irregularity-narrowing in the right vertebral artery.

months after the initial event by her own choice. She remained clinically asymptomatic.

In patients with SCAD, $70 \%$ patients had extracoronary vascular involvement, and of these, FMD was the most common vascular abnormality occurring in $80 \%$ of subjects [3]. Given that histological diagnosis is rarely made, we hope that future angiographic definition of FMD will consider incorporating SCAD in the addition to simultaneous dissection in other extra-cardiac vessels without other characteristic findings currently included in angiographic criteria.

Drs. Calenda and Olin refer to genetic analysis demonstrating association between cervical artery dissection and rs9349379[G] allele located on chromosome 6 in the phosphatase and actin regulator 1 gene (PHACTR1) $[4,5]$. These studies were performed on Finnish, non-Finnish Europeans and US patients of European origin.

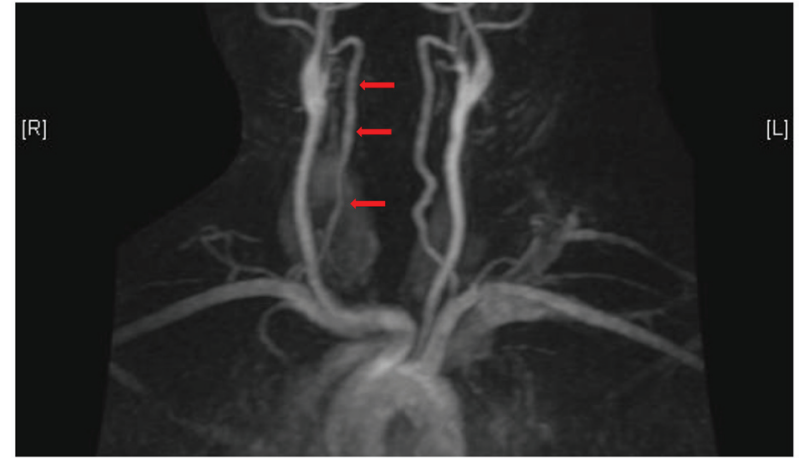

Figure 3. Follow-up imaging, 15 months after initial presentation. Healed dissection areas in the left vertebral artery with worsening diffuse narrowing in the right vertebral artery.

Our patient is African-American and is likely of distinct ancestral origin compared to Finnish and non-Finnish Europeans. Presence of similar genetic associations between different races is possible; however, assumptions of the same genetic susceptibility cannot be inferred without further analysis.

Tannaz Shoja, MD

Craig Basman, MD

Suresh Jain, MD

Aditya Mangla, MD

Zoran Lasic, MD

\section{References}

1. Shoja T, Basman C, Jain S, Mangla A, Lasic Z. Postpartum sudden cardiac death after spontaneous coronary artery dissection in a patient with fibromuscular dysplasia. Cardiol Res. 2017;8(6):327-330.

2. Plouin PF, Baguet JP, Thony F, Ormezzano O, Azarine A, Silhol F, Oppenheim C, et al. High prevalence of multiple arterial bed lesions in patients with fibromuscular dysplasia: the ARCADIA registry (assessment of renal and cervical artery dysplasia). Hypertension. 2017;70(3):652658.

3. Prasad M, Tweet MS, Hayes SN, Leng S, Liang JJ, Eleid MF, Gulati R, et al. Prevalence of extracoronary vascular abnormalities and fibromuscular dysplasia in patients with spontaneous coronary artery dissection. Am J Cardiol. 2015;115(12):1672-1677.

4. Debette S, Kamatani Y, Metso TM, Kloss M, Chauhan G, Engelter ST, Pezzini A, et al. Common variation in PHACTR1 is associated with susceptibility to cervical artery dissection. Nat Genet. 2015;47(1):78-83.

5. Kiando SR, Tucker NR, Castro-Vega LJ, Katz A, D'Escamard V, Treard C, Fraher D, et al. PHACTR1 is a genetic susceptibility locus for fibromuscular dysplasia supporting its complexgenetic pattern of inheritance. PLoS Genet. 2016;12(10):e1006367. 